\title{
MEASUREMENT OF CHROMATIC EFFECTS IN LEP
}

\author{
D. Brandt, P. Castro, K. Cornelis, A. Hofmann, G. Morpurgo, G.L. Sabbi and A. Verdier, \\ CERN, CH-1211 Geneva 23, Switzerland
}

\begin{abstract}
The chromaticity produced by the insertions and the lattice of LEP is corrected in the FODO lattice of the arcs which has horizontal and vertical phase advances of $90^{\circ}$ and $60^{\circ}$ for the LEP optics presently used. This is done with two horizontal and three vertical sextupole families. To check this correction scheme the non-linear chromaticities have been measured by observing the betatron tunes as a function of RF-frequency, i.e. of momentum deviation. The differences between the results and the expected effects of the sextupoles are interpreted in terms of octupole and decapole components in the dipole magnets. To check the distribution around the ring of the chromaticities and their corrections the betatron phase advances have been measured as a function of momentum deviation. This was done by observing an excited betatron oscillation for 1024 turns in all beam position monitors for different RF-frequencies. The results clearly show the negative chromaticity produced in the straight sections and its correction in the arcs. The parasitic sextupole components are found to be spread uniformly in the arcs within the measurement errors. Under this condition one finds that they have a negligible effect on the dynamic aperture.
\end{abstract}

\section{INTRODUCTION}

The non-linear chromaticity of the LEP machine at CERN is corrected with sextupole families. For the optics used from 1993 to 1995 , there are five sextupole families. This number is linked with the phase advances in the arc cells where the sextupoles are located. Three families are used for the vertical plane where the phase advance is $60^{\circ}$ and two families are used for the horizontal plane where the phase advance is $90^{\circ}$. Detailed specifications concerning sextupole families are given in another paper at this conference [1]. The basic motivation to use more than two sextupole families is to compensate the off-momentum mismatch of the low- $\beta$ insertions. For instance if the chromaticity is corrected with two sextupole families in the arcs only, the off-momentum betatron motion becomes unstable for a momentum deviation of about 0.003 .

Due to the dependence of the radiation damping partitions on $\Delta p$, the measurement of the variation of the tunes with momentum can only be used to check the chromaticity correction in a small range of momentum deviations. This is still sufficient to validate the correction done. However what has not been done up to now is to measure the distribution of the parasitic sextupole, octupole and decapole components in the dipoles of the arc cells. Although it should be possible to predict the chromaticity and the tune dependence on amplitude from the known average value of the sextupole and octupole components, it is interesting to check this and see that the distribution of these components in the arc cells is smooth enough not to make higher order effects. This is what has been done and is reported here.

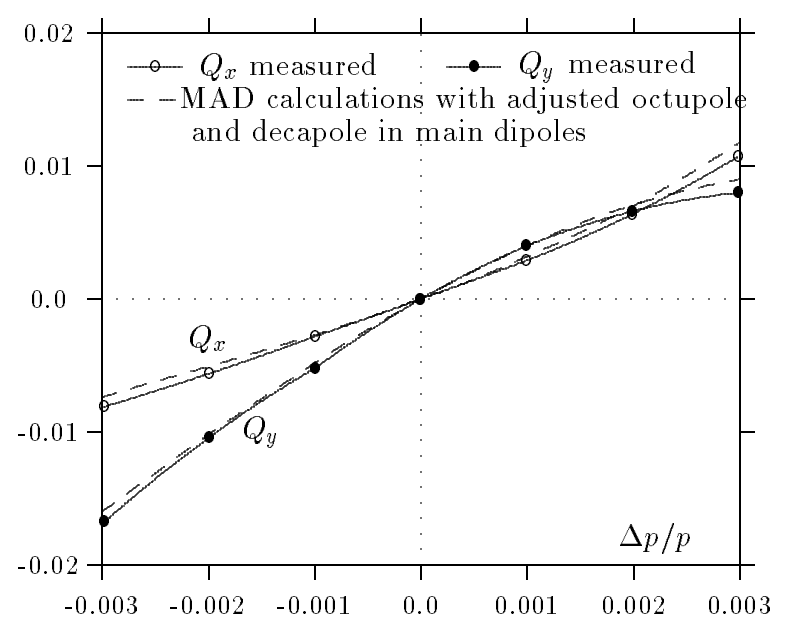

Figure. 1. Measured and calculated tunes vs. $\Delta p / p$

\section{MEASUREMENT OF THE NON-LINEAR CHROMATICITY BY CHANGING THE RF FREQUENCY.}

This method is very well known. For a given value of the RF frequency, there is a momentum of the beam such that its centre of gravity corresponds to the synchronous particle. The momentum deviation $\Delta p / p$ from the central value and the relative $\mathrm{RF}$ frequency change $\frac{\Delta f_{R F}}{f_{R F}}$ are related by :

$$
\frac{\Delta f_{R F}}{f_{R F}}=\left(\frac{1}{\gamma^{2}}-\alpha_{c}\right) \frac{\Delta p}{p}
$$

where $\alpha_{c}$ is the momentum compaction factor.

In the case of LEP some care has to be taken for such a measurement because of the tune change due to the SPS pulsing. In order to eliminate it, a phase-lock loop (PLL) is used to record permanently the machine tunes [2]. By inspecting this record, it is easy to distinguish peaks due to the SPS pulsing and to take the right tune value outside of the perturbed areas. The accuracy of this measurement itself is better than the LEP stability (power supplies and mechanical stability), it is of the order of 0.0001 . In order to eliminate tune drifts due to the LEP stability, the PLL record is done while the RF frequency is incremented and decremented by the same value. This makes it possible to obtain an accuracy of the tune measurement of about 0.0002 . Given this high accuracy, a correction of the measurement due to the change of the bunch length with $\Delta p / p$ and due to the coupling has been investigated [3]. It was found that a small correction has to be applied to the value of the first derivative of the tunes but no correction from these effects have to be applied to the higher order derivatives as they are well below the measurement errors. The corrected values of the tunes versus momentum deviation are shown in Fig. 1. If the measured octupole and decapole errors in 
the dipoles are used directly, a large discrepancy between measurement and calculation would occur. To reduce it to the small values shown in Fig. 1 these components had to be multiplied by large factors of -45 and 630 respectively. This corresponds to a total relative field error of about 0.007 , which should have showed up in the magnetic measurements. On the other hand the measurement of the chromatic phase advance shown below rules out non-uniform sextupole errors which could produce higher order effects. Thus we are left with a large discrepancy not yet understood.

\section{MEASUREMENT OF THE CHROMATIC PHASE ADVANCE}

\section{A. Introduction}

The dependence of the betatron tunes on momentum is called "Q-prime" and is given by $Q^{\prime}=d Q /(d p / p)$. Sometimes the above quantities are divided by the tune and called chromaticities. Since the quadrupole focusing strength decreases with energy, the normal focusing structure produces a negative contribution to the chromaticity. This has to be corrected with sextupole magnets located at finite dispersion. In LEP the eight long straight sections containing the interaction regions have vanishing dispersion and do not contain sextupoles. They give negative contributions to the chromaticities. The sextupoles in the eight arcs have a strength adjusted to compensate the negative chromaticity contributions of the focusing structures in the arc itself and in the long straight sections. This results in a local over compensation of the chromaticity.

To describe these local effects we use the dependence of the phase advance on momentum deviation [4], [5] which we will call chromatic phase advance

$$
\frac{d \mu_{x}}{d p / p}, \frac{d \mu_{y}}{d p / p} \text { or normalized: } \frac{d \mu_{x} / 2 \pi}{d p / p}, \frac{d \mu_{y} / 2 \pi}{d p / p}
$$

The integral over the latter quantities around the ring gives again $Q^{\prime}$. A measurement of the chromatic phase advance is an excellent check of the chromaticity and its correction in the ring.

\section{B. Measurement method}

The phase advance is measured at the beam position monitors (BPM) using the "1000-turns" system [6]. With this system the beam position is recorded for 1024 revolutions $i$ at each BPM $k$ while one specific bunch is excited in one plane (horizontal or vertical) with the betatron tune frequency by the tunemeasurement shaker [2]. The amplitude of the oscillations depends on the proximity of the exciting frequency to the tune and on the strength of the excitation. In order to extract the amplitude $A$ and the phase $\phi$ of the betatron oscillations, a harmonic fit is made through the 1024 beam position measurement $\left\{x_{i}\right\}_{i=1}^{1024}$ of each BPM $k$ with the known frequency of excitation

$$
C_{k}=\sum_{i=1}^{N} x_{i} \cos \left(2 \pi Q_{x} i\right) \quad, \quad S_{k}=\sum_{i=1}^{N} x_{i} \sin \left(2 \pi Q_{x} i\right)
$$

to obtain

$$
A_{k}=\frac{2 \sqrt{C_{k}^{2}+S_{k}^{2}}}{N} \quad, \quad \phi_{k}=-\arctan \left(\frac{S_{k}}{C_{k}}\right)
$$

where $N=1024$ is the number of samples. $\phi_{k}$ corresponds to the phase advance $\mu_{x}$ at the BPM $k$ plus an arbitrary constant for all BPM. For precise phase measurements, the beam must be excited to high amplitude in order to achieve the highest signalto-noise ratio. Typical oscillation amplitudes are in the order of a few millimeters. The error of the phase is [7]

$$
\sigma_{\mu}=\frac{1}{A} \sqrt{\frac{2}{N}} \sigma_{x}
$$

with $\sigma_{x}$ being the beam position error. The phase error is typically about 2-3 mrad for a signal of $2 \mathrm{~mm}$ of amplitude (4 mm peak-to-peak) and 1024 points.

\section{The experiment}

A positron beam of about $0.1 \mathrm{~mA}$ per bunch was accumulated on the nominal RF-frequency with the injection optics K21P20 which has detuned low-beta insertions. The horizontal and vertical phase advances where measured in all the BOM monitors using the "1000-turn" system described above for the RF-frequency deviations of $-100 \mathrm{~Hz}, 0 \mathrm{~Hz}$, and $+100 \mathrm{~Hz}$. The corresponding energy deviations are obtained with the relation (1) and are listed together with the measured tunes in Table 1. For each measurement the phase advance calculated with MAD [8] for the central orbit was subtracted. All the measurements used for analysis were taken with about the same betatron oscillation amplitude to avoid effects of non-linearities.

\begin{tabular}{|l|l|l|l|}
\hline$\Delta f_{R F}$ & $\Delta E / E$ & $Q_{x}$ & $Q_{y}$ \\
\hline$-100 \mathrm{~Hz}$ & 0.00153 & 0.255 & 0.219 \\
$0 \mathrm{~Hz}$ & 0.0 & 0.255 & 0.219 \\
$100 \mathrm{~Hz}$ & -0.00153 & 0.255 & 0.217 \\
\hline
\end{tabular}

Table I

Parameters of the phase advance measurements

\section{Analysis of the measured data}

A first look at the experimental results indicated that some selection of the data had to be made. A few monitors gave values which were far outside the range of the remaining ones. The corresponding data were ignored. This was the case for 8 monitors in the horizontal plane and for 6 monitors in the vertical plane. Since the absolute phase is not known, the average phase of all the accepted monitors was set to zero for each measurement. For each monitor a linear fit through the three points taken at different energy deviations was made and the quantity $(d \mu / 2 \pi) /(d p / p)$ calculated.

\section{E. Results}

The measured normalized phase advance per relative energy change and the corresponding calculations with MAD are plotted in Fig. 2 against the normalized phase. Clearly visible are the chromaticity contributions of the different parts. The four straight sections containing interaction points IP2, IP4, IP6, and IP8 give large negative contributions of about $\Delta Q_{x}=-16.4$ and $\Delta Q_{y}^{\prime}=-12.1$ each, being within $4 \%$ of the calculated values. The other straight sections have smaller effects $\Delta Q_{x}^{\prime}=-7.7$ 

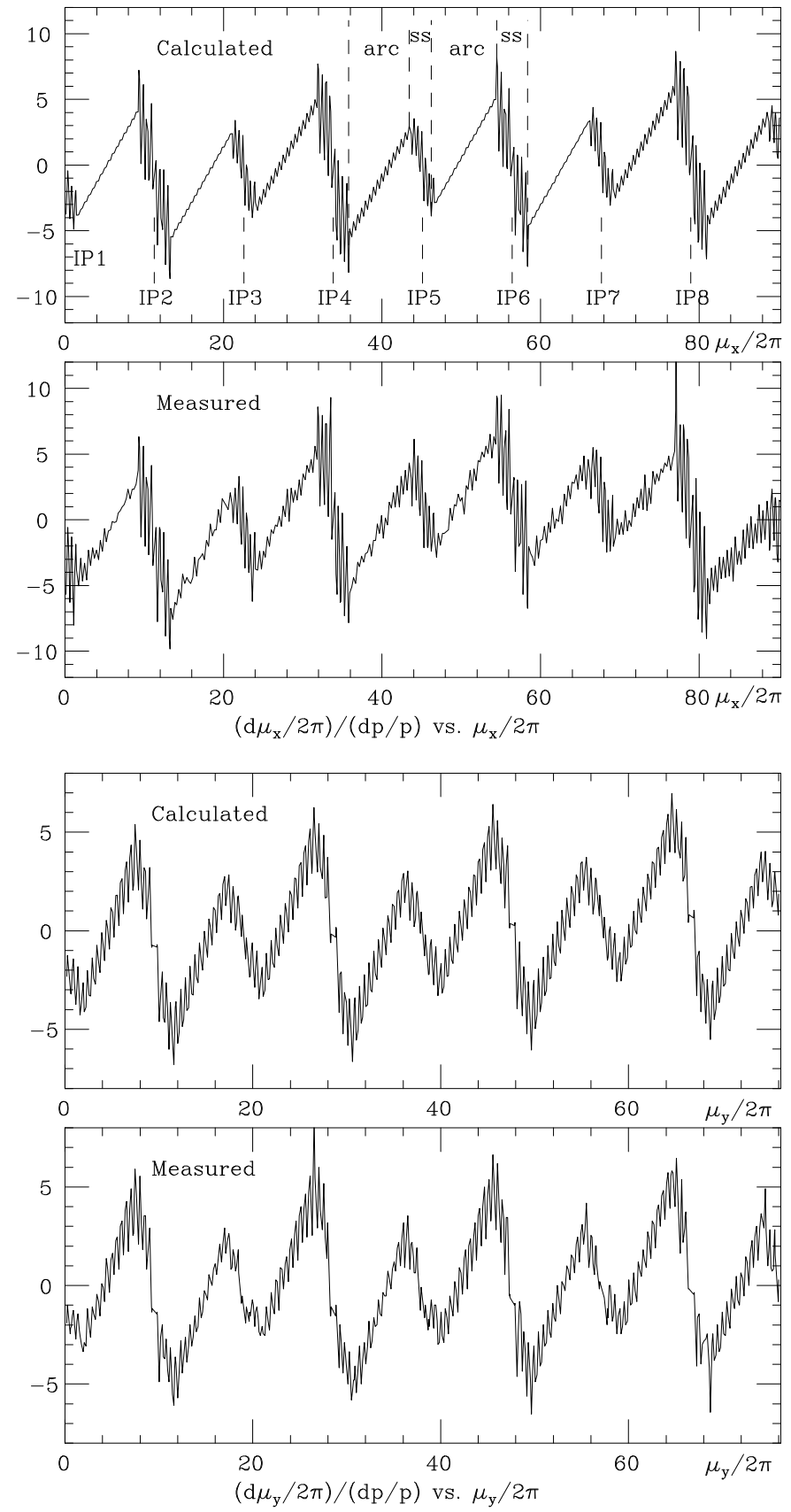

Figure. 2. Chromatic phase advances ('ss': straight sections)

and $\Delta Q_{y}^{\prime}=-6.1$ each, which agree within $12 \%$ with expectations. The measured variation of the individual sections is about $10 \%$. Also the positive contributions of the arcs containing the sextupoles are clearly visible. We measure $\Delta Q_{r}^{\prime}=8.6$ and $\Delta Q_{y}^{\prime}=8.5$ per octant. The horizontal value is about $6 \%$ larger than expected whereas the vertical one agrees within $1 \%$. The variation of the individual octants is smaller than $9 \%$ for $Q_{x}^{\prime}$ and smaller than $2 \%$ for $Q_{y}^{\prime}$. All these discrepancies are probably due to measurement errors with the exception that the absolute values of the individual horizontal contributions are in average about $5 \%$ too large. Strong localized sextupole errors can be ruled out by these measurements.

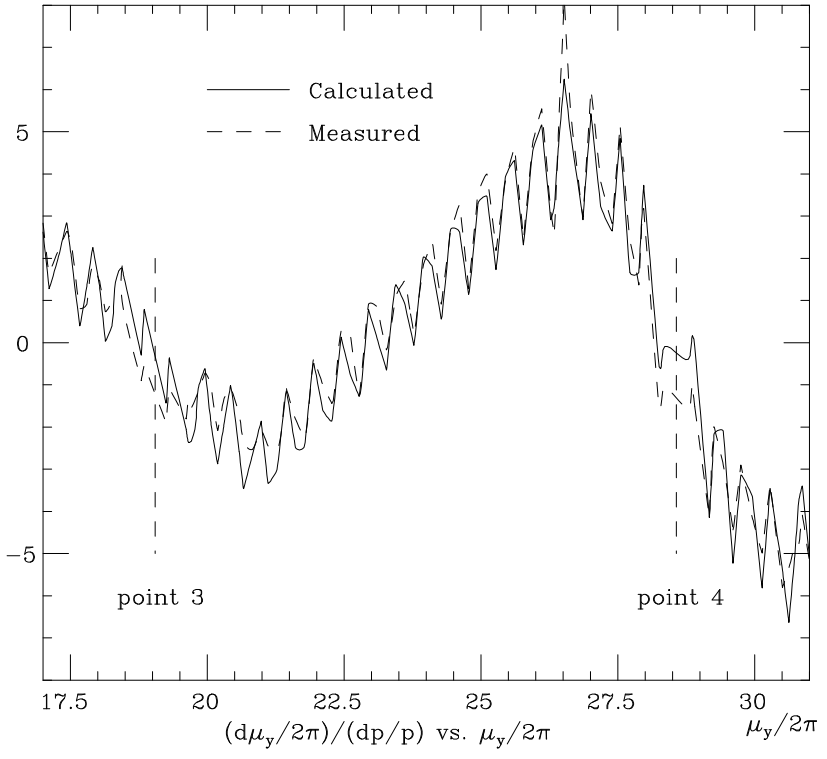

Figure. 3. Details of the calculated and measured vertical chromatic phase advance

In general the agreement between experiment and calculation is very good not only for the global behavior of the chromatic phase advance but also for its details as shown in Fig. 3. The fast oscillation of the calculated and measured chromatic phase advance is due to a mismatch of the off-energy optics resulting in a beating both of the beta function and the phase advance. As expected this beating goes with twice the betatron phase advance.

\section{References}

[1] A. Verdier "Chromaticity correction with sextupole families", This conference.

[2] K.D.Lohmann, M. Placidi and H. Schmickler; "Design and Functionality of the LEP Q-meter"; Proc. of the 2nd European Part. Accel. Conf., EPAC; Nice, June 1990, p. 774.

[3] A. Verdier, "Measurement of the non-linear chromaticity at 46 GeV (G05P46hv2)", CERN SL-MD Note 117, 1994.

[4] H. Zyngier; "Strategy for Chromaticity Correction", Orsay LAL Note 77/35, 1977.

[5] B.W. Montague; 'Linear Optics for Improved Chromaticity Correction"; LEP note 165, 1979.

[6] J. Borer, C. Bovet, A. Burns and G. Morpurgo; "Harmonic Analysis of Coherent Bunch Oscillations In LEP"; Proc. of the 3rd European Part. Accel. Conf., EPAC; Berlin, March 1992, p. 1082.

[7] A. Burns, P. Castro, G. Morpurgo and R. Schmidt, "Betatron Function Measurement at LEP Using the BOM 1000 Turns Facility"; Proc. of the 1993 IEEE Part. Accel. Conf., PAC; Washington, 17-20 May 1993, p. 2103.

[8] H. Grote, F.C. Iselin "The Mad Program"; CERN/SL/90-13. 